\title{
Gut microbiome alterations in type 1 autoimmune pancreatitis after induction of remission by prednisolone
}

\author{
Ken Kamata ${ }^{1}$, Tomohiro Watanabe ${ }^{1}$, Kosuke Minaga ${ }^{1}$, Akane Hara ${ }^{1}$, Ikue Sekai ${ }^{1}$, Yasuo \\ Otsuka $^{1}$, Tomoe Yoshikawa ${ }^{1}$, Ah-Mee Park ${ }^{1}$, and Masatoshi Kudo ${ }^{1}$ \\ ${ }^{1}$ Kindai University Graduate School of Medical Sciences
}

May 26, 2020

\begin{abstract}
Although increasing evidence demonstrates the association between intestinal dysbiosis and pancreatic diseases, such as chronic pancreatitis and pancreatic cancer, it remains largely unknown whether intestinal dysbiosis is involved in the immunopathogenesis of autoimmune pancreatitis (AIP). Recently, we found that intestinal dysbiosis mediates experimental AIP via the activation of plasmacytoid dendritic cells (pDCs), which can produce IFN- $\alpha$ and IL-33. However, candidate pathobionts for type 1 AIP have not been identified. In this study, we tried to identify pathobionts associated with type 1 AIP. Fecal samples were obtained from type 1 AIP patients before and after prednisolone (PSL) treatment and subjected to 16S ribosomal RNA sequencing to evaluate the composition of intestinal bacteria. Induction of remission by PSL was associated with the complete disappearance of Klebsiella species from feces, in two of the three analyzed patients with type 1 AIP. To assess the pathogenicity of Klebsiella species, mild experimental AIP was induced in MRL/MpJ mice by repeated injections of $10 \mu \mathrm{g}$ of polyinosinic-polycytidylic acid (poly (I:C)) in combination with oral administration of heat-killed Klebsiella pneumoniae. The AIP pathology score was significantly higher in MRL/MpJ mice that received both oral administration of heat-killed K. pneumoniae and intraperitoneal injections of poly (I:C) than in those administered with either agent alone. Pancreatic accumulation of pDCs capable of producing large amounts of IFN- $\alpha$ and IL-33 was also significantly higher in mice that received both treatments. These data suggest that intestinal colonization by K. pneumoniae may play a pathogenic role in AIP.
\end{abstract}

\section{Abbreviations used in this paper}

Ab, antibody; Ag, antigen; AIP, autoimmune pancreatitis; ELISA, enzyme-linked immunosorbent assay; GI, gastrointestinal; H\&E, hematoxylin and eosin; IgG4-RD, IgG4-related disease; IP, intraperitoneal; K. pneumoniae, Klebsiella pneumoniae ;Klebsiella spp. , Klebsiella species ; MAMP, microbe-associated molecular pattern; OTU, operational taxonomic unit; pDC, plasmacytoid dendritic cell; PMNCs, pancreatic mononuclear cells; poly (I:C), polyinosinic-polycytidylic acid; PSC, primary sclerosing cholangitis; PSL, prednisolone; rRNA, ribosomal RNA; TLR, toll-like receptor.

\section{Introduction}

The human gastrointestinal (GI) tract is colonized by more than 100 trillion of microorganisms [1-3]. A large number of diverse microbial species residing in the GI tract play a major role in multiple aspects of host physiology, including the maturation of the systemic and mucosal immune responses, and production and absorption of nutrients [1-3]. There is growing evidence supporting the concept that compositional and functional alterations of the gut microbiome, called intestinal dysbiosis, are associated with the pathogenesis of not only the GI tract but also that of extra-GI disorders [1-3]. Moreover, recent studies have highlighted the importance of intestinal dysbiosis in the development of several pancreatic diseases, such as acute pancreatitis, chronic pancreatitis, and pancreatic cancer $[4,5]$. 
Type 1 autoimmune pancreatitis (AIP) is a pancreatic manifestation of systemic IgG4-related disease (IgG4$\mathrm{RD}$ ), a newly established disease entity [6-8]. AIP and IgG4-RD are diagnosed based on elevated serum concentrations of IgG4 antibodies (Abs) and massive infiltration of IgG4-expressing plasma cells into the affected organs [6-8]. Although the immunopathogeneses of AIP and IgG4-RD have been poorly understood, recent identification of candidate autoantigens (autoAgs) strongly suggests that these disorders are driven by excessive adaptive immunity against such target antigens (Ags) [9-11]. Indeed, effector T cell subpopulations, such as $\mathrm{T}$ helper type 2 cells, regulatory $\mathrm{T}$ cells, and follicular helper $\mathrm{T}$ cells, are involved in the generation of IgG4 Ab responses [6]. In addition to the demonstration of the role of adaptive immune responses, studies have provided evidence that innate immunity also affects the development of AIP and IgG4-RD [12-17]. For example, enhanced production of the innate immunity cytokines type I IFN and IL-33 is a prominent feature of experimental AIP and human IgG4-RD [14, 16-19]. Moreover, stimulation of peripheral blood monocytes and basophils isolated from patients with type 1 AIP promotes IgG4 Ab production by B cells isolated from healthy controls in the presence of microbe-associated molecular patterns (MAMPs) [12, 13]. Pathogenic roles played by toll-like receptors (TLRs) recognizing MAMPs have been also implicated because several TLRs are expressed at higher levels in the pancreas and salivary glands of patients with IgG4-RD [15, 17].

Although disturbances in innate immunity underlie the immunopathogenesis of AIP and IgG4-RD, it remains largely unknown whether intestinal dysbiosis promotes the pathogenic innate immune responses characterizing these disorders. We recently provided evidence that intestinal dysbiosis increases the sensitivity to experimental AIP through the activation of type I IFN and IL-33-mediated signaling pathways [20]; however, the role of intestinal dysbiosis in human AIP has not been clarified. In this study, we explored the compositional changes in fecal microbiota in samples obtained from patients with type 1 AIP, before and after treatment with prednisolone (PSL), and found that the induction of remission might be associated with complete disappearance ofKlebsiella species (spp). Thereafter, we determined the pathogenicity of Klebsiella pneumoniae (K. pneumoniae) in murine experimental AIP.

\section{Methods}

\section{Patients and fecal microbiota analysis}

Three AIP patients that met the diagnostic criteria for type 1 AIP [21] were enrolled in this study. They were treated with PSL for the induction of remission. Oral administration of PSL at an initial dose of $0.6-0.7 \mathrm{mg} / \mathrm{kg}$ followed by scheduled tapering regimen ( $5 \mathrm{mg}$ every two weeks) successfully induced clinical remission in all patients, as evaluated by the disappearance of diffuse pancreatic swelling and reduction in serum IgG4 concentrations. The study protocol conformed to the ethical guidelines for human clinical research established by the Japanese Ministry of Health, Labor, and Welfare and was approved by the ethics committee at the Kindai University Faculty of Medicine. Written informed consent was obtained from all patients during enrollment.

Fecal samples were obtained from all three patients before, as well as two months after, the PSL treatment. Fecal microbiota analysis was performed as described previously [20, 22]. DNA samples extracted from the stool were subjected to polymerase chain reaction for the amplification of $16 \mathrm{~S}$ ribosomal RNA (16S rRNA) V3 and V4 regions, followed by sequencing using the MIseq system (Illumina Japan, Tokyo, Japan). Sequence data were processed by Trimmomatic, Cutadapt, and Fastq-join programs; subsequently, operational taxonomic units (OTUs) were determined by using QIIME programs.

\section{Preparation of heat-killed $K$. pneumoniae}

K. pneumoniae was purchased from ATCC (Manassas, Virginia) and grown overnight in brain heart infusion (BHI) broth (Eiken chemical, Tochigi, Japan) at $37^{\circ} \mathrm{C}$. The bacterial number was determined by the absorbance at $600 \mathrm{~nm}\left(0.1=1 \times 10^{8} / \mathrm{ml}\right)$. K. pneumoniae was subjected to incubation at $70^{\circ} \mathrm{C}$ for 30 minutes to generate heat-killed $K$. pneumoniae .

\section{Induction of experimental AIP}

Experimental AIP was induced as previously described [14, 16, 20]. Briefly, MRL/MpJ mice (Japan SLC, 
Hamamatsu, Japan) were treated with an intraperitoneal (IP) injection of poly (I:C) (10 $\mu$ g; InvivoGen, San Diego, CA) twice a week, for a total of 16 times to induce mild AIP [20]. Other groups of MRL/MpJ mice received an oral administration of $K$. pneumoniae $\left(2 \times 10^{7} /\right.$ each mouse) and/or an IP injection of $10 \mu \mathrm{g}$ of polyinosinic-polycytidylic acid (poly (I:C)) twice a week, for a total of 16 times. Ethical permission for the animal experiments was obtained from the Review Boards of Kindai University Faculty of Medicine.

\section{Histological analysis}

The mice were sacrificed 3 hours after the final injection of poly (I:C) or oral administration of K. pneumoniae . Pancreatic samples were subjected to fixation with $10 \%$ formalin followed by hematoxylin and eosin (H\&E) staining; subsequently, pathological assessment was done by using a scoring system for AIP in accordance with previous reports $[14,16,20]$. Pancreas inflammation was scored as follows: 0, pancreas without mononuclear cell infiltration; 1, mononuclear cell aggregation and/or infiltration within the interstitium without parenchymal destruction; 2, focal parenchymal destruction with mononuclear cell infiltration; 3, diffuse parenchymal destruction but with retention of some intact parenchymal areas; 4, destruction of almost all pancreatic tissue, except for the pancreatic islets, or its replacement with fibrosis or adipose tissue $[14,16,20]$. At least two histological microscopy images (Biozero, BZ-8100, Keyence) were captured from each slide.

\section{Isolation of pancreatic mononuclear cells and flow cytometry analysis}

Pancreatic mononuclear cells (PMNCs) were isolated from mouse pancreas in accordance with previous reports [23]. PMNCs were stained with FITC or PE-conjugated anti-B220 Ab (eBioscience, San Diego, CA), anti-pDC Ag-1 (PDCA-1) Ab (eBioscience), anti-CD3 Ab (eBioscience), anti-CD11b Ab (eBioscience), or anti-CD11c Ab (eBioscience) and then subjected to flow cytometric analysis using an Accuri C6 flow cytometer (BD Biosciences, San Jose, CA) and CFlow Plus software (BD Biosciences).

\section{Cytokine assays}

Cytokine concentrations in the pancreas were measured by the enzyme-linked immunosorbent assay (ELISA), as previously described $[24,25]$. Concentrations of IFN- $\alpha$, IFN- $\gamma$, IL-6, IL-13, IL-33, and TNF- $\alpha$ were determined using ELISA kits obtained from R\&D Systems (Minneapolis, MN) and eBioscience.

\section{Statistical analysis}

The Student's $t$-test was used to evaluate the significance of differences. Statistical analysis was performed using Prism (GraphPad Software, La Jolla, CA, USA). Differences were considered statistically significant if $P<0.05$.

\section{Results}

In our previous reports, we showed that intestinal dysbiosis mediates the development of experimental AIP by activating pDCs, which have the ability to produce large amounts of IFN- $\alpha$ and IL-33 [6, 14, 16, 19, 20]. This study aimed to clarify alterations in gut microbiota that have been poorly defined in type 1 AIP patients.

\section{Case presentation of type 1 AIP and IgG4-RD}

Three cases with type 1 AIP with solitary involvement of the pancreas were enrolled in this study (Fig. 1A). All patients met the diagnostic criteria for definite type 1 AIP [21]. All patients were treated with PSL; then, remission was successfully induced, as evidenced by reductions in serum concentrations of IgG4 (Fig. 1B). In a typical case of type 1 AIP (Case 1), the diffuse pancreatic swelling and capsule-like rim seen before PSL administration disappeared after the treatment (Fig. 1C).

\section{Fecal microbiota diversity}

To assess the effects of PSL treatment on intestinal microbiota in type 1 AIP, we performed next generation sequencing of $16 \mathrm{~S}$ rRNA in fecal samples, as previously described [20, 22]. Fecal microbial diversity was 
comparable before and after PSL treatment, as judged by the rarefaction analysis of alpha diversity (Fig. 1D) and by the Shannon index (data not shown).

\section{Alternations in fecal microbiota composition}

Next, we assessed alterations in fecal microbiota composition in type 1 AIP following PSL treatment. Analysis at the order level revealed that induction of remission by PSL treatment was accompanied by the disappearance of Enterobacteriales and Fusobacterialesfrom the gut of two and one of the three AIP patients, respectively (Fig. 2, 3).

Relative abundance of each bacteria at the genus level is shown in Fig. 4A. Although the composition of fecal microbiota did not differ significantly between active and remitted diseases at the genus level, the following trends were seen in several bacterial species. Colonization by Klebsiella spp. was detected in two of the three patients before treatment with PSL. Surprisingly, clinical remission induced by PSL was accompanied by complete disappearance ofKlebsiella spp. in two of the three patients (Cases 1 and 2, Fig. 4B). These data were consistent with complete disappearance ofEnterobacteriales at the order level in two of three paints after PSL treatment. In contrast, the relative abundance of Ruminococcus spp. was higher in the feces of all patients after treatment (Fig. 4A, B). A marked reduction in colonization of Fusobacterium spp.after PSL treatment was seen in one patient (Case 3) who did not harborKlebsiella spp. before PSL treatment. AlthoughBifidobacterium spp. were highly abundant in the feces of mice exhibiting AIP in our previous study [20], the relative abundance ofBifidobacterium spp. was comparable before and after the treatment in this study. Thus, induction of remission by PSL was accompanied by complete disappearance of Klebsiella spp. from the feces of two type 1 AIP patients and by a marked reduction inFusobacterium spp. from those of one patient who did not harbor the former bacterium. These fecal microbiota data suggest that colonization by Klebsiella spp. might be involved in the development of type 1 AIP in patients who harbor this bacterium.

\section{Pathogenicity of heat-killed $K$. pneumoniae in experimental AIP}

The association between reduced colonization by Klebsiella spp.and induction of remission led us to examine the pathogenicity of this bacterium in AIP. Therefore, we employed a well-established murine model of AIP in MRL/MpJ mice $[14,16,19,20]$. Repeated IP injections of poly (I:C) into MRL/MpJ mice at a higher dose of $100 \mu \mathrm{g}$ triggered severe AIP, characterized by massive infiltration of immune cells, destruction of acinar architecture, and fibrosis; whereas, repeated IP injections at a lower dose of $10 \mu \mathrm{g}$ caused mild AIP with infiltration of immune cells $[14,16,19,20]$. We hypothesized that immune responses against Klebsiella $s p p$. increase the sensitivity to experimental AIP. To test this hypothesis, MRL/MpJ mice received combined treatment with orally administered heat-killed $K$. pneumoniae and IP injections of poly (I:C) (10 $\mu \mathrm{g})$ twice a week, for a total of 16 times.

As expected, infiltration of immune cells was seen in the pancreas of MRL/MpJ mice that received IP injections of $10 \mu \mathrm{g}$ of poly (I:C) alone (Fig. 5A). Interestingly, this infiltration was accompanied by the destruction of acinar architecture in the pancreas of MRL/MpJ mice following combined treatment with orally administered heat-killed $K$. pneumoniae and IP injections of poly (I:C) (Fig. 5A). Quantitative assessment of pathological scores confirmed that oral administration of heat-killed K. pneumoniae promoted the development of pancreatitis (Fig. 5B).

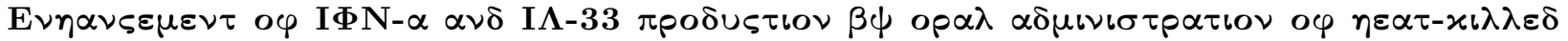 K. $\pi \nu \epsilon \nu \mu о \nu \imath \alpha \epsilon$}

Experimental AIP induced by repeated IP injections of poly (I:C) depends on the activation of pDCs, which produce large amounts of IFN- $\alpha$ and IL-33 [14, 16, 19, 20]. Therefore, we examined whether exacerbation of AIP by orally administered $K$. pneumoniae was associated with the activation of pDCs producing both IFN- $\alpha$ and IL-33. We examined pancreatic immune cell populations by flow cytometry. The percentage of pDCs defined as PDCA-1+B220 ${ }^{\text {low }}$ cells was significantly higher in MRL/MpJ mice that received orally administered heat-killed K. pneumoniae and IP injections of poly (I:C) than in those treated with the 
bacterium or IP injections of poly (I:C) alone (Fig. 6A, B). In contrast, the combined treatment with orally administered heat-killed $K$. pneumoniae and IP injections of poly (I:C) did not change the percentages of

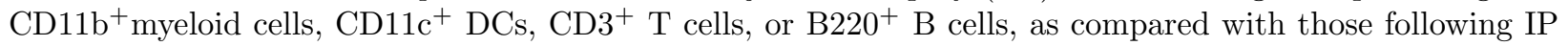
injections of poly (I:C) alone (Fig. 6A, B).

Lastly, we determined profiles of proinflammatory cytokine responses in the pancreas. Consistent with marked accumulation of $\mathrm{pDCs}$, co-administration of heat-killed $K$. pneumoniae and poly (I:C) markedly increased production of IFN- $\alpha$ and IL-33 in the pancreas, as compared with their levels after the administration of heat-killed $K$. pneumoniae or poly (I:C) alone (Fig. 7). Pancreatic levels of the prototypical innate immunity cytokines IL-6 and TNF- $\alpha$ induced by poly (I:C) injections were similar in the presence and absence of co-administered heat-killed K. pneumonia. We previously showed that pancreatic pDCs producing both IFN- $\alpha$ and IL-33 induce pancreatic expression of adaptive immunity cytokines, including pro-inflammatory IFN- $\gamma$ and pro-fibrogenic IL-13 [14, 16, 20]. In line with the results of those previous studies, co-administration of heat-killed K. pneumoniae and poly (I:C) enhanced pro-inflammatory and profibrogenic adaptive immune responses because co-administration markedly elevated pancreatic production of IFN- $\gamma$ and IL-13, as compared with the effect of poly (I:C) administration alone (Fig. 7). Collectively, these data suggest that exacerbation of experimental AIP by oral administration of heat-killed K. pneumoniae was accompanied by pancreatic accumulation of pDCs producing IFN- $\alpha$ and IL-33.

\section{Discussion}

Although intestinal dysbiosis is recognized as one of the pathogenic factors for the development of several pancreatic diseases, such as chronic alcoholic pancreatitis and pancreatic cancer $[4,5]$, pathogenic roles of intestinal dysbiosis in AIP have remained poorly defined. In our previous studies, we found that intestinal dysbiosis mediates experimental AIP through the activation of $\mathrm{pDCs}$, which subsequently produce large amounts of IFN- $\alpha$ and IL-33 [20]. In this study, we demonstrated differences in the composition of fecal microbiota in samples from three type 1 AIP patients, at active and remitted phases. Interestingly, induction of clinical remission by PSL was accompanied by complete disappearance of Klebsiella spp. from the gut of two patients who harbored this bacterium before the treatment. We then explored the pathogenicity of Klebsiella spp.in experimental AIP by utilizing MRL/MpJ mice treated with $10 \mu \mathrm{g}$ of poly (I:C). In contrast to the manifestations in the well-established severe AIP model induced by repeated IP injections of $100 \mu \mathrm{g}$ of poly (I:C), repeated IP injections of poly (I:C) at a tenfold lower dose $(10 \mu \mathrm{g})$ into MRL/MpJ mice resulted in the development of mild AIP [20]. By utilizing this mild AIP model, we found that the degree of AIP was greater in mice treated with both oral administration of heat-killed $K$. pneumoniae and IP injections of 10 $\mu \mathrm{g}$ of poly (I:C) than in those that received either treatment alone. Moreover, oral administration of heatkilled K. pneumoniae combined with IP injections of poly (I:C) promoted pancreatic accumulation of pDCs; consequently, this elevated IFN- $\alpha$ and IL-33 production by those cells. Thus, moderate AIP mediated by the pancreatic accumulation of pDCs was successfully induced in MRL/MpJ mice only when oral administration of heat-killed K. pneumoniae was combined with IP injections of poly (I:C). Therefore, oral administration of heat-killed $K$. pneumoniae increased the sensitivity to poly (I:C)-induced experimental AIP. Together with the complete disappearance of Klebsiella spp.from feces of type 1 AIP patients at the remission phase, these data strongly suggest that immune responses against Klebsiella spp.may be involved in the development of type 1 AIP.

Induction of remission by PSL led to complete disappearance ofKlebsiella spp. from the feces of two of the three type 1 AIP patients. In contrast, colonization by Ruminococcus spp. was promoted after PSL treatment in all of the three patients. Thus, this study suggests that gut colonization by Klebsiella spp. andRuminococcus spp. may play pro-inflammatory and anti-inflammatory roles, respectively, in type 1 AIP. Such increased abundance ofKlebsiella spp. and decreased abundance of Ruminococcus spp. have been observed in the feces of patients who developed pancreatic fistula after pancreaticoduodenectomy for pancreatic cancer [26]. In addition, fecal samples obtained from chronic alcoholic pancreatitis patients exhibited higher abundance of Klebsiella spp. than fecal samples from patients with alcoholic hepatitis [27]. These reports and the present study support the view thatKlebsiella spp. may function as a pathobiont for 
$\mathrm{CP}$ and AIP as in the case of primary sclerosing cholangitis (PSC) [28]. However, it should be noted that our sample size was too small to establish with certainty gut microbial alterations in type 1 AIP before and after PSL treatment. Moreover, pathogenic immune responses to colonization by Klebsiella spp. might be masked by the differences in microbiota profiles, as the proportions of Bacteroides, Streptococcus, andClostridium $s p p$. were reported to be lower in patients with AIP than in those with CP [29].

Oral administration of heat-killed K. pneumoniae increased the sensitivity to experimental AIP induced by low doses of poly (I:C) in this study. Thus, immune responses activated by heat-killed K. pneumoniae alone were not sufficient for the induction of experimental AIP. As for the pathogenic intestinal bacteria other than K. pneumoniae, Haruta et al. reported that repeated IP injections of heat-killed Escherichia coli led to the development of experimental AIP in C57BL/6 mice [30]. They further identified FliC, one of the flagella subunit proteins of this bacterium, as a bacterial pathogenic factor because repeated IP injections of FliC alone caused AIP in C57BL/6 mice [31]. Therefore, persistent exposure to FliC derived from $E$. coli is sufficient for the development of experimental AIP in C57BL/6 mice. These previous studies utilizing heat-killed E. coli and C57BL/6 mice differed from our present data in that the development of AIP in MRL/MpJ mice requires administration not only of heat-killed K. pneumoniae but also of poly (I:C) at low doses. Whether persistent exposure to heat-killed intestinal bacteria plays a critical or supportive role in the development of AIP remains to be elucidated. Nonetheless, those studies and our present data indicate that the development of immune reactions against intestinal bacteria may affect AIP.

One question arising from the present study is how heat-killed $K$. pneumoniae activates pDCs, which produce large amounts of IFN- $\alpha$ and IL-33. In this regard, host defense against pulmonary infection with $K$. pneumoniae depends upon TLR9-mediated proinflammatory cytokine responses [32]. Moreover, the depletion of pDCs impairs proinflammatory cytokine responses in mice during sublethal pulmonary infection with K. pneumoniae [33]. Given the fact that TLR9 activation is a strong inducer of IFN- $\alpha$ production in pDCs $[34,35]$, it is possible that heat-killed $K$. pneumoniae directly stimulates IFN- $\alpha$ production by pDCs through TLR9 activation. This idea is supported by the fact that activation of type I IFN signaling pathways, and subsequent production of C-X-C motif chemokine ligand 10, mediate host defense against murine $K$. pneumoniae pneumonia [36, 37].

Nakamoto et al. identified K. pneumoniae as one of gut pathobionts for PSC [28]. Colonization by K. pneumoniaederived from PSC patients into gnotobiotic mice led to the development of hepatobiliary injury through the induction of $\mathrm{CD} 4^{+} \mathrm{T}$ cells, which produced IFN- $\gamma$ and IL-17 [28]. Colonization by K. pneumoniae increased the sensitivity to experimental PSC, as the development of chronic hepatobiliary inflammation required both inoculation of this bacterium and oral administration of 3,5-dicarbethoxy-1,4-dihydrocollidine, the latter of which triggered hepatobiliary injury. Together with our findings, those data suggest that immune responses against $K$. pneumoniae may promote the development of AIP and PSC in the presence of potent triggers. It should be noted, however, that AIP and PSC are completely different in terms of the sensitivity to PSL treatment in that most of patients with type 1 AIP respond to PSL treatment while those of PSC do not. Therefore, other factors than the pathogenic colonization of Klebsiella spp. might be involved in the different pathophysiology between these immune disorders.

In conclusion, colonization by Klebsiella spp. may mediate the development of type 1 AIP through the activation of pDCs.Klebsiella spp. may be a type 1 AIP pathobiont. However, a confirmation of this hypothesis awaits future studies in which gut microbiome will have to be studied in a large number of type 1 AIP patients.

\section{Acknowledgements}

This work was supported by Grants-in-Aid for Scientific Research (19K08455, 19K17506, 20K16975) from the Japan Society for the Promotion of Science, Takeda Science Foundation, Smoking Research Foundation, Yakult Bio-Science Foundation, SENSHIN Medical Research Foundation, and Japan Agency for Medical Research and Development (AMED) for Research on Intractable Diseases.

\section{Disclosures}


The authors declare that they have no conflict of interest.

\section{Author contributions}

K.K, T.W., K.M., A.H, and A.P. designed and performed the experiments. K.K., K.M., and A.H. collected patient samples. K.K., T.W., K.M., A.H., I.S., and Y.O. analyzed the data. K.K., T.W., K.M., A.P. and M.K. prepared the manuscript.

\section{Figure legends}

\section{Figure 1. Characteristics of patients with type 1 autoimmune pancreatitis.}

Three patients with type 1 autoimmune pancreatitis were enrolled in this study. They were treated with prednisolone (PSL) for the induction of remission. (A) Patient age, sex, and affected organs.(B) Serum concentrations of IgG4 before and after PSL treatment. (C) Contrast-enhanced computed tomography images of case 1 before (left) and after (right) PSL treatment. (D) Fecal samples obtained from all the patients were subjected to $16 \mathrm{~S}$ ribosomal RNA sequencing to evaluate the composition of intestinal bacteria. Alpha rarefaction plots. Species richness expressed as the number of operational taxonomic units at increasing sequencing depth is illustrated. Results are expressed as the mean + standard error.

Figure 2. Gut microbiome alterations in patients with type 1 autoimmune pancreatitis before and after prednisolone treatment.Fecal samples were subjected to $16 \mathrm{~S}$ ribosomal RNA sequencing to evaluate the composition of intestinal bacteria. A total of six samples were obtained from three patients with type 1 autoimmune pancreatitis before and after prednisolone (PSL) treatment. Relative bacterial abundance at the order level is shown. Each bar shows relative bacterial abundance in each patient before and after PSL treatment.

Figure 3. Reduction of Enterobacteriales in patients with type 1 autoimmune pancreatitis after prednisolone treatment. A total of six samples were obtained from three patients with type 1 autoimmune pancreatitis before and after prednisolone (PSL) treatment. Fecal samples were subjected to $16 \mathrm{~S}$ ribosomal RNA sequencing to evaluate the composition of intestinal bacteria. Alterations in gut microbiota, at the order level, are shown for each patient.

Figure 4. Reduction of Klebsiella species in patients with type 1 autoimmune pancreatitis after prednisolone treatment. A total of six samples were obtained from three patients with type 1 autoimmune pancreatitis before and after prednisolone (PSL) treatment. Fecal samples were subjected to $16 \mathrm{~S}$ ribosomal RNA sequencing to evaluate the composition of intestinal bacteria. (A)Relative abundance of a broad range of intestinal bacteria is shown at the genus level. Results are expressed as the mean + standard error in three samples before (black bar) and after (white bar) PSL treatment. (B) Alterations in gut microbiota, at the genus level, are shown for each patient.

Figure 5. Increased sensitivity to polyinosinic-polycytidylic acid-induced experimental autoimmune pancreatitis in MRL/MpJ mice orally administered with heat-killed Klebsiella pneumoniae. MRL/MpJ mice were injected with polyinosinic-polycytidylic acid (poly (I:C), $10 \mu \mathrm{g}$ ), orally administered heat-killed $K$. pneumoniae, received both these treatments twice a week for a total of 16 times, or left untreated. Mice were sacrificed $3 \mathrm{~h}$ after the final injection of poly (I:C) or oral adiministration of heat-killed K. pneumoniae .(A) Pancreatic tissues were stained with hematoxylin and eosin (H\&E). Representative $\mathrm{H} \& \mathrm{E}$ images of the four experimental groups are shown (magnification $400 \times$ ). (B) Pathology scores as assessed by $\mathrm{H} \& \mathrm{E}$ staining ( $n=5$ for all four experimental groups). Results are expressed as the mean + standard error. ${ }^{*} P<0.05$, as compared with mice treated with poly (I:C) alone.

Figure 6. Pancreatic accumulation of plasmacytoid dendritic cells in MRL/MpJ mice that received oral administration of heat-killedKlebsiella pneumoniae and intraperitoneal injections of polyinosinic-polycytidylic acid. MRL/MpJ mice were injected with polyinosinic-polycytidylic acid (poly (I:C), $10 \mu \mathrm{g}$ ), orally administered heat-killed K. pneumoniae, received both these treatments twice a week for a total of 16 times, or left untreated. (A, B)Pancreatic mononuclear cells were analyzed by 
flow cytometry to evaluate the population of plasmacytoid dendritic cell antigen $1(\mathrm{PDCA}-1)^{+} \mathrm{B} 220^{\text {low }}$ plasmacytoid dendritic cells (pDCs), CD11b ${ }^{+}$myeloid cells, CD11c ${ }^{+} \mathrm{DCs}, \mathrm{CD}^{+} \mathrm{T}$ cells, and $\mathrm{B} 220^{+} \mathrm{B}$ cells $\left(n=5\right.$ for all four experimental groups). Results are expressed as the mean + standard error. ${ }^{* *} P<$ 0.01, as compared with mice treated with poly (I:C) alone.

Figure 7. Pancreatic expression of proinflammatory cytokines in MRL/MpJ mice that received oral administration of heat-killedKlebsiella pneumoniae and intraperitoneal injections of polyinosinic-polycytidylic acid. MRL/MpJ mice were injected with polyinosinic-polycytidylic acid (poly (I:C), $10 \mu \mathrm{g}$ ), orally administered heat-killed K. pneumoniae, received both these treatments twice a week for a total of 16 times, or left untreated. Pancreatic levels of pro-inflammatory cytokines were determined by the enzyme-linked immunosorbent assay ( $n=5$ for all four experimental groups). Results are expressed as the mean + standard error. ${ }^{* *} P<0.01$, as compared with mice treated with poly (I:C) alone.

\section{References}

1. Skelly AN, Sato Y, Kearney S, Honda K. Mining the microbiota for microbial and metabolite-based immunotherapies. Nat Rev Immunol 2019; $19: 305-23$.

2. Lynch SV, Pedersen O. The Human Intestinal Microbiome in Health and Disease. N Engl J Med 2016; $375: 2369-79$.

3. Levy M, Kolodziejczyk AA, Thaiss CA, Elinav E. Dysbiosis and the immune system. Nat Rev Immunol 2017; $17: 219-32$.

4. Akshintala VS, Talukdar R, Singh VK, Goggins M. The Gut Microbiome in Pancreatic Disease. Clin Gastroenterol Hepatol 2019;17:290-5.

5. Adolph TE, Mayr L, Grabherr F, Schwarzler J, Tilg H. Pancreas-Microbiota Cross Talk in Health and Disease. Annu Rev Nutr 2019; 39 :249-66.

6. Watanabe T, Minaga K, Kamata K, Kudo M, Strober W. Mechanistic Insights into Autoimmune Pancreatitis and IgG4-Related Disease.Trends Immunol 2018; 39 :874-89.

7. Kamisawa T, Zen Y, Pillai S, Stone JH. IgG4-related disease.Lancet 2015; 385 :1460-71.

8. Stone JH, Zen Y, Deshpande V. IgG4-related disease. N Engl J Med 2012; 366 :539-51.

9. Hubers LM, Vos H, Schuurman AR, Erken R, Oude Elferink RP, Burgering B, van de Graaf SFJ, Beuers U. Annexin A11 is targeted by IgG4 and IgG1 autoantibodies in IgG4-related disease. Gut 2018;67 :728-35.

10. Shiokawa M, Kodama Y, Sekiguchi K, Kuwada T, Tomono T, Kuriyama K, Yamazaki H, Morita T, Marui S, Sogabe Y, Kakiuchi N, Matsumori T, Mima A, Nishikawa Y, Ueda T, Tsuda M, Yamauchi Y, Sakuma Y, Maruno T, Uza N, Tsuruyama T, Mimori T, Seno H, Chiba T. Laminin 511 is a target antigen in autoimmune pancreatitis. Sci Transl Med 2018; 10 .

11. Perugino CA, AlSalem SB, Mattoo H, Della-Torre E, Mahajan V, Ganesh G, Allard-Chamard H, Wallace Z, Montesi SB, Kreuzer J, Haas W, Stone JH, Pillai S. Identification of galectin-3 as an autoantigen in patients with IgG4-related disease. J Allergy Clin Immunol 2019;143 :736-45 e6.

12. Watanabe T, Yamashita K, Fujikawa S, Sakurai T, Kudo M, Shiokawa M, Kodama Y, Uchida K, Okazaki K, Chiba T. Activation of Toll-like Receptors and NOD-like Receptors Is Involved in Enhanced IgG4 Responses in Autoimmune Pancreatitis. Arthritis \& Rheumatism 2012;64 :914-24.

13. Watanabe T, Yamashita K, Sakurai T, Kudo M, Shiokawa M, Uza N, Kodama Y, Uchida K, Okazaki K, Chiba T. Toll-like receptor activation in basophils contributes to the development of IgG4-related disease.J Gastroenterol 2013; $48: 247-53$.

14. Arai Y, Yamashita K, Kuriyama K, Shiokawa M, Kodama Y, Sakurai T, Mizugishi K, Uchida K, Kadowaki N, Takaori-Kondo A, Kudo M, Okazaki K, Strober W, Chiba T, Watanabe T. Plasmacytoid Dendritic 
Cell Activation and IFN-alpha Production Are Prominent Features of Murine Autoimmune Pancreatitis and Human IgG4-Related Autoimmune Pancreatitis. J Immunol 2015; 195 :3033-44.

15. Fukui Y, Uchida K, Sakaguchi Y, Fukui T, Nishio A, Shikata N, Sakaida N, Uemura Y, Satoi S, Okazaki K. Possible involvement of Toll-like receptor 7 in the development of type 1 autoimmune pancreatitis. J Gastroenterol 2015; 50 :435-44.

16. Watanabe T, Yamashita K, Arai Y, Minaga K, Kamata K, Nagai T, Komeda Y, Takenaka M, Hagiwara S, Ida H, Sakurai T, Nishida N, Strober W, Kudo M. Chronic Fibro-Inflammatory Responses in Autoimmune Pancreatitis Depend on IFN-alpha and IL-33 Produced by Plasmacytoid Dendritic Cells. J Immunol 2017; $198: 3886-96$.

17. Ishiguro N, Moriyama M, Furusho K, Furukawa S, Shibata T, Murakami Y, Chinju A, Haque A, Gion Y, Ohta M, Maehara T, Tanaka A, Yamauchi M, Sakamoto M, Mochizuki K, Ono Y, Hayashida JN, Sato Y, Kiyoshima T, Yamamoto H, Miyake K, Nakamura S. Activated M2 Macrophages Contribute to the Pathogenesis of IgG4-Related Disease via Toll-like Receptor 7/Interleukin-33 Signaling. Arthritis Rheumatol $2020 ; 72: 166-78$.

18. Furukawa S, Moriyama M, Miyake K, Nakashima H, Tanaka A, Maehara T, Iizuka-Koga M, Tsuboi H, Hayashida JN, Ishiguro N, Yamauchi M, Sumida T, Nakamura S. Interleukin-33 produced by M2 macrophages and other immune cells contributes to Th2 immune reaction of IgG4-related disease.Sci Rep $2017 ; 7: 42413$.

19. Minaga K, Watanabe T, Arai Y, Shiokawa M, Hara A, Yoshikawa T, Kamata K, Yamashita K, Kudo M. Activation of interferon regulatory factor 7 in plasmacytoid dendritic cells promotes experimental autoimmune pancreatitis. J Gastroenterol 2020;55 :565-76.

20. Kamata K, Watanabe T, Minaga K, Hara A, Yoshikawa T, Okamoto A, Yamao K, Takenaka M, Park AM, Kudo M. Intestinal dysbiosis mediates experimental autoimmune pancreatitis via activation of plasmacytoid dendritic cells. Int Immunol 2019; 31 :795-809.

21. Kawa S, Kamisawa T, Notohara K, Fujinaga Y, Inoue D, Koyama T, Okazaki K. Japanese Clinical Diagnostic Criteria for Autoimmune Pancreatitis, 2018: Revision of Japanese Clinical Diagnostic Criteria for Autoimmune Pancreatitis, 2011. Pancreas 2020;49 :e13-e4.

22. Okamoto K, Watanabe T, Komeda Y, Okamoto A, Minaga K, Kamata K, Yamao K, Takenaka M, Hagiwara S, Sakurai T, Tanaka T, Sakamoto H, Fujimoto K, Nishida N, Kudo M. Dysbiosis-Associated Polyposis of the Colon-Cap Polyposis. Front Immunol 2018; 9 :918.

23. Kamata K, Watanabe T, Minaga K, Strober W, Kudo M. Autoimmune Pancreatitis Mouse Model. Curr Protoc Immunol 2018;120:15 31 1-15 318.

24. Watanabe T, Minaga K, Kamata K, Sakurai T, Komeda Y, Nagai T, Kitani A, Tajima M, Fuss IJ, Kudo M, Strober W. RICK/RIP2 is a NOD2-independent nodal point of gut inflammation. Int Immunol $2019 ; 31: 669-83$.

25. Watanabe T, Sadakane Y, Yagama N, Sakurai T, Ezoe H, Kudo M, Chiba T, Strober W. Nucleotidebinding oligomerization domain 1 acts in concert with the cholecystokinin receptor agonist, cerulein, to induce IL-33-dependent chronic pancreatitis. Mucosal Immunol 2016;9 :1234-49.

26. Rogers MB, Aveson V, Firek B, Yeh A, Brooks B, Brower-Sinning R, Steve J, Banfield JF, Zureikat A, Hogg M, Boone BA, Zeh HJ, Morowitz MJ. Disturbances of the Perioperative Microbiome Across Multiple Body Sites in Patients Undergoing Pancreaticoduodenectomy. Pancreas 2017;46 :260-7.

27. Ciocan D, Rebours V, Voican CS, Wrzosek L, Puchois V, Cassard AM, Perlemuter G. Characterization of intestinal microbiota in alcoholic patients with and without alcoholic hepatitis or chronic alcoholic pancreatitis. Sci Rep 2018; $8: 4822$. 
28. Nakamoto N, Sasaki N, Aoki R, Miyamoto K, Suda W, Teratani T, Suzuki T, Koda Y, Chu PS, Taniki N, Yamaguchi A, Kanamori M, Kamada N, Hattori M, Ashida H, Sakamoto M, Atarashi K, Narushima S, Yoshimura A, Honda K, Sato T, Kanai T. Gut pathobionts underlie intestinal barrier dysfunction and liver T helper 17 cell immune response in primary sclerosing cholangitis. Nat Microbiol 2019; 4 :492-503.

29. Hamada S, Masamune A, Nabeshima T, Shimosegawa T. Differences in Gut Microbiota Profiles between Autoimmune Pancreatitis and Chronic Pancreatitis. Tohoku J Exp Med 2018; 244 :113-7.

30. Haruta I, Yanagisawa N, Kawamura S, Furukawa T, Shimizu K, Kato H, Kobayashi M, Shiratori K, Yagi J. A mouse model of autoimmune pancreatitis with salivary gland involvement triggered by innate immunity via persistent exposure to avirulent bacteria. Lab Invest 2010; 90 :1757-69.

31. Yanagisawa N, Haruta I, Shimizu K, Furukawa T, Higuchi T, Shibata N, Shiratori K, Yagi J. Identification of commensal flora-associated antigen as a pathogenetic factor of autoimmune pancreatitis.Pancreatology $2014 ; 14: 100-6$.

32. Bhan U, Lukacs NW, Osterholzer JJ, Newstead MW, Zeng X, Moore TA, McMillan TR, Krieg AM, Akira $\mathrm{S}$, Standiford TJ. TLR9 is required for protective innate immunity in Gram-negative bacterial pneumonia: role of dendritic cells. J Immunol 2007; 179 :3937-46.

33. Lippitsch A, Baal N, Chukovetskyi Y, Cunningham S, Michel G, Dietert K, Gurtner C, Gruber AD, Bein G, Hackstein H. Plasmacytoid dendritic cell depletion modifies FoxP3+ T cell homeostasis and the clinical course of bacterial pneumonia in mice. J Leukoc Biol 2019;106 :977-85.

34. Swiecki M, Colonna M. The multifaceted biology of plasmacytoid dendritic cells. Nat Rev Immunol $2015 ; 15: 471-85$.

35. Gilliet M, Cao W, Liu YJ. Plasmacytoid dendritic cells: sensing nucleic acids in viral infection and autoimmune diseases. Nat Rev Immunol 2008; 8 :594-606.

36. Ivin M, Dumigan A, de Vasconcelos FN, Ebner F, Borroni M, Kavirayani A, Przybyszewska KN, Ingram RJ, Lienenklaus S, Kalinke U, Stoiber D, Bengoechea JA, Kovarik P. Natural killer cell-intrinsic type I IFN signaling controls Klebsiella pneumoniae growth during lung infection. PLoS Pathog 2017; 13 :e1006696.

37. Zeng X, Moore TA, Newstead MW, Deng JC, Kunkel SL, Luster AD, Standiford TJ. Interferon-inducible protein 10, but not monokine induced by gamma interferon, promotes protective type 1 immunity in murine Klebsiella pneumoniae pneumonia. Infect Immun 2005;73 :8226-36. 
A

\begin{tabular}{|l|c|c|c|}
\hline & Age & M/F & Affected organ \\
\hline Case 1 & 54 & M & Pancreas \\
\hline Case 2 & 70 & M & Pancreas \\
\hline Case 3 & 46 & M & Pancreas \\
\hline
\end{tabular}

C

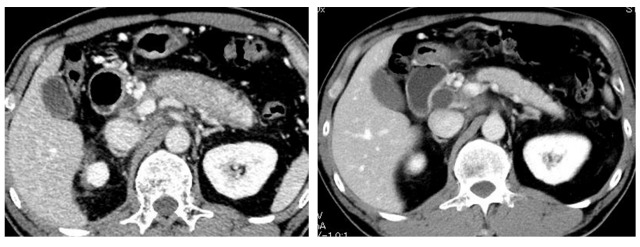

B

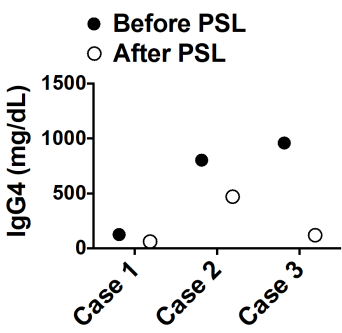

D

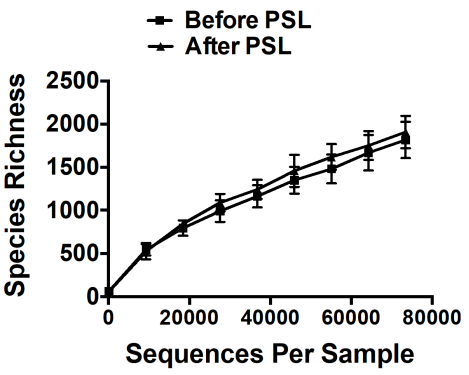

Figure 1
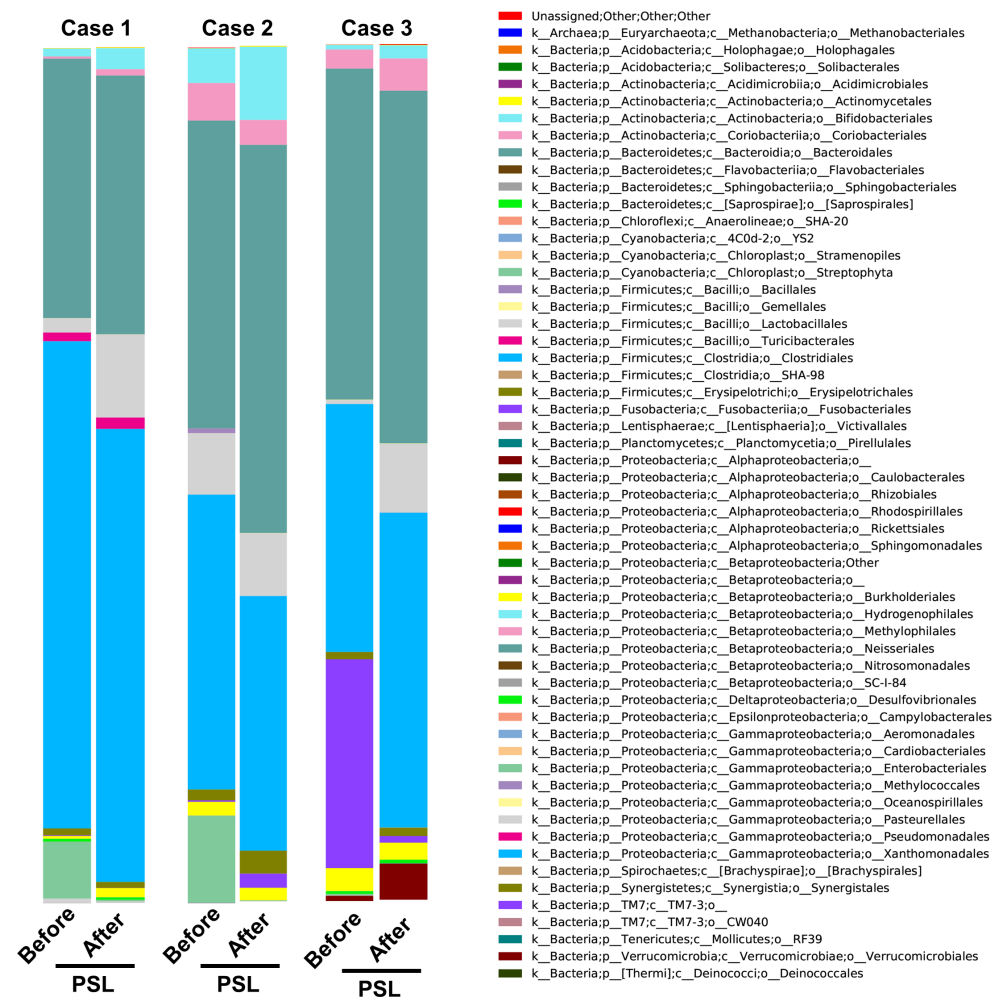

Figure 2 

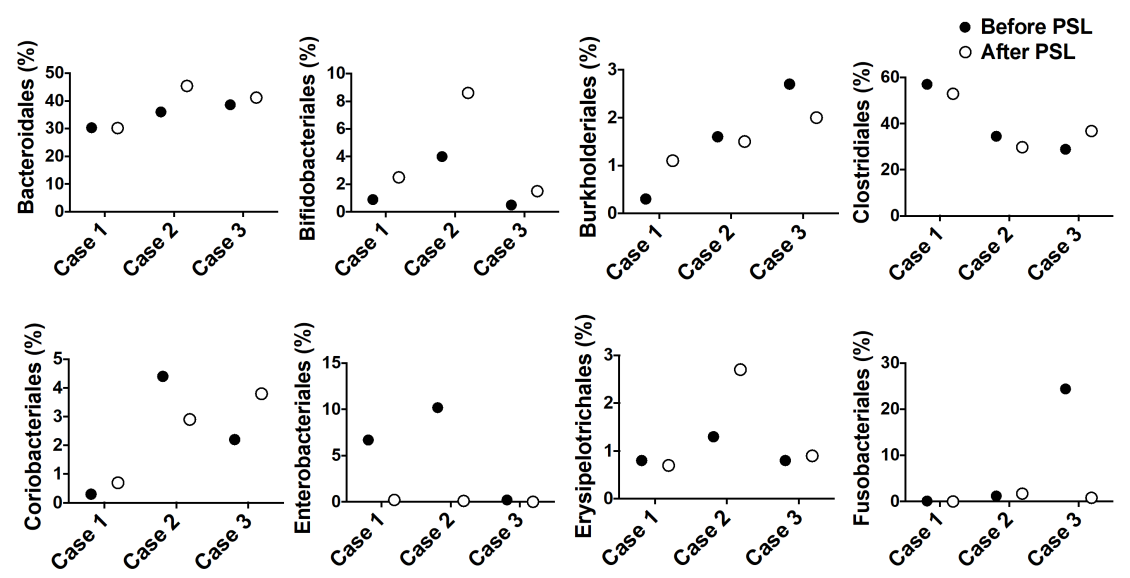

Figure 3

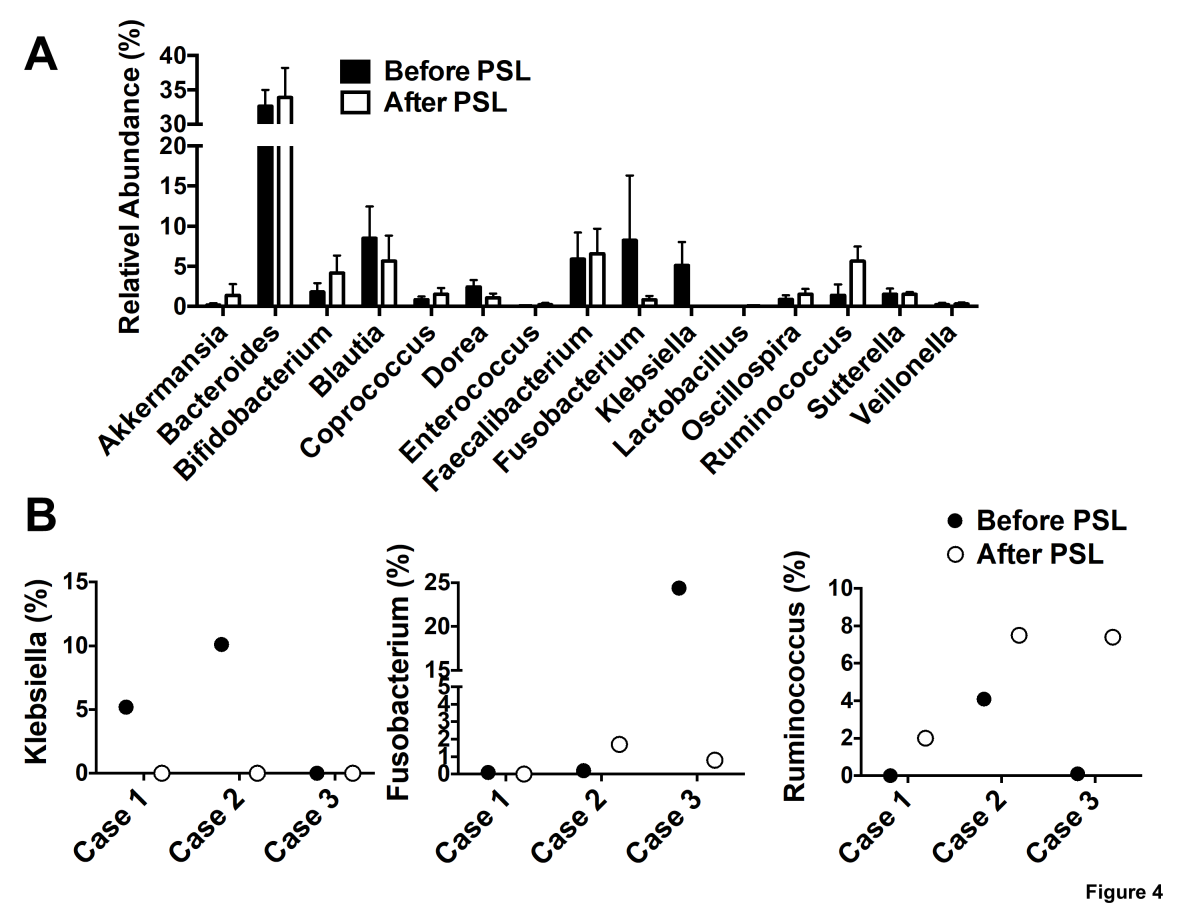


A
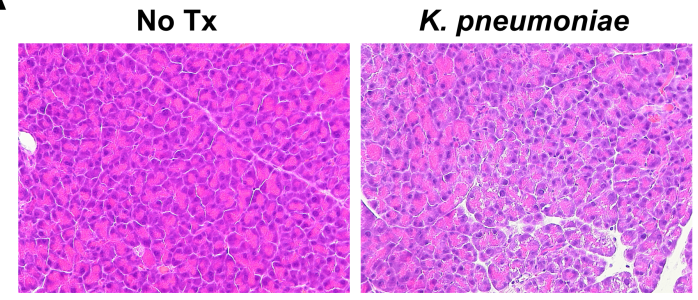

Poly (I:C)

Poly (I:C)

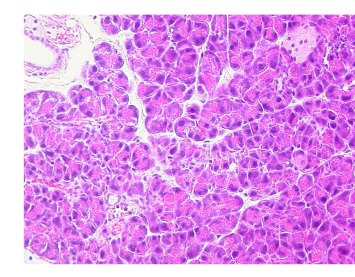

K. pneumoniae

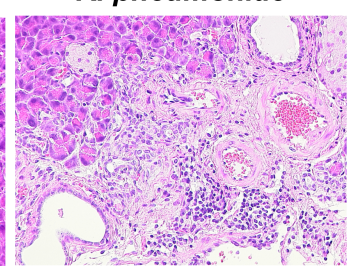

B

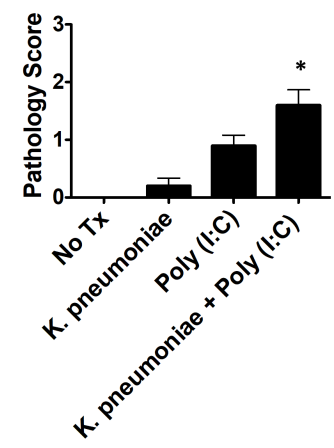

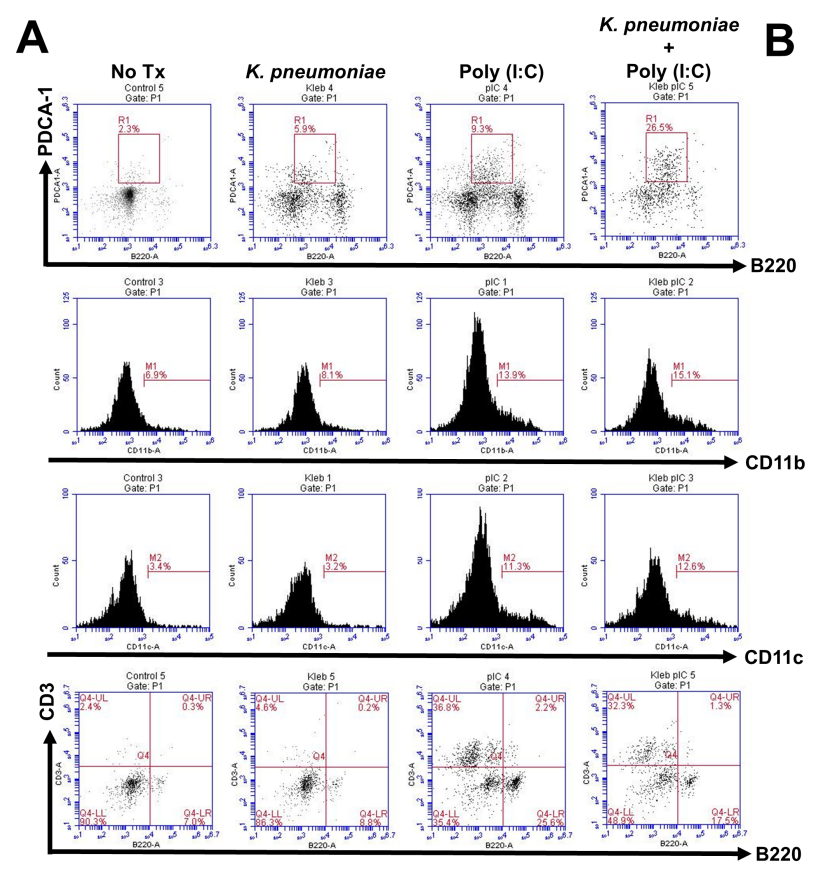

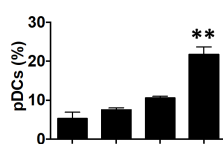

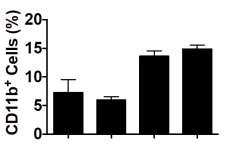

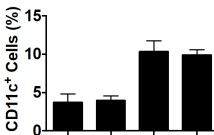

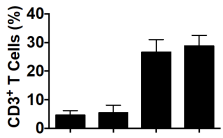

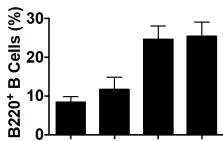
60

Figure 6 

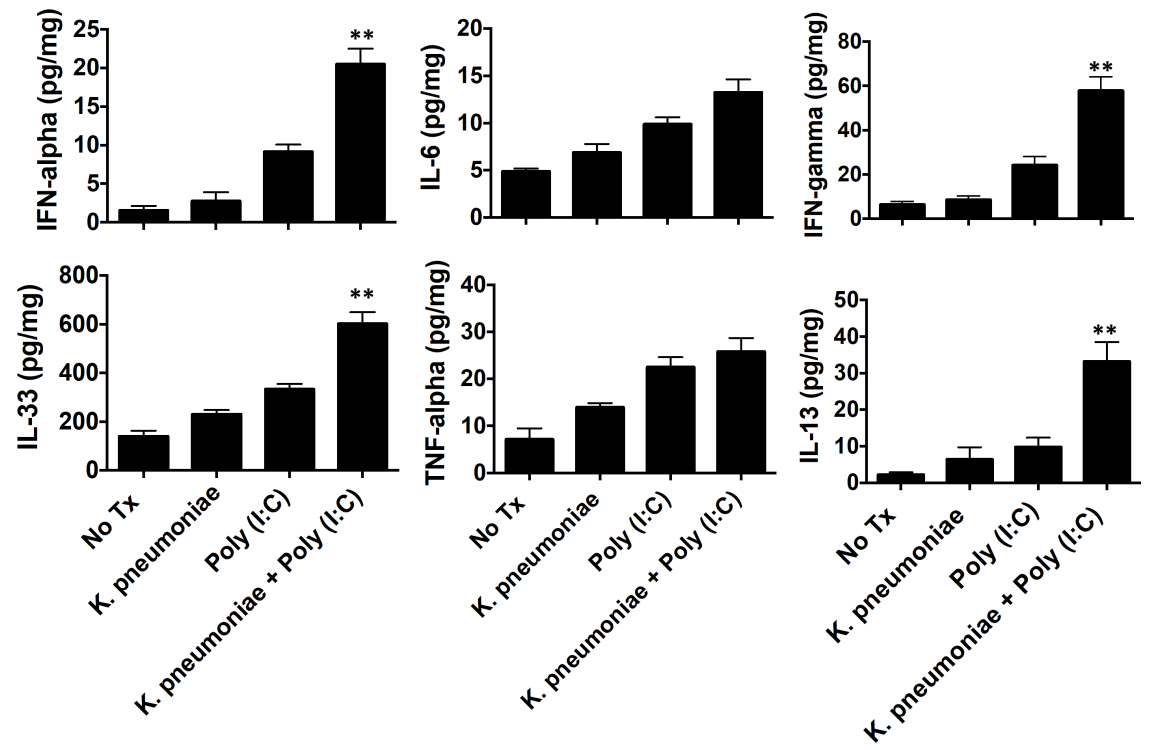

Figure 7 